\title{
High-Order Time-Accurate Parallel Schemes for Parabolic Singularly Perturbed Problems with Convection*
}

\author{
P. W. Hemker, Amsterdam, G. I. Shishkin and L. P. Shishkina, Ekaterinburg
}

Received December 3, 1999; revised April 20, 2000

\begin{abstract}
The first boundary value problem for a singularly perturbed parabolic equation of convection-diffusion type on an interval is studied. For the approximation of the boundary value problem we use earlier developed finite difference schemes, $\varepsilon$-uniformly of a high order of accuracy with respect to time, based on defect correction. New in this paper is the introduction of a partitioning of the domain for these $\varepsilon$-uniform schemes. We determine the conditions under which the difference schemes, applied independently on subdomains may accelerate ( $\varepsilon$-uniformly) the solution of the boundary value problem without losing the accuracy of the original schemes. Hence, the simultaneous solution on subdomains can in principle be used for parallelization of the computational method.
\end{abstract}

AMS Subject Classifications: 65N22, 65N55, 35K20, 35B25, 35A40.

Key Words: Parabolic PDEs, convection-diffusion, higher-order time-accuracy schemes, defect correction, $\varepsilon$-uniform convergence, parallel algorithms, Schwarz-like methods.

\section{Introduction}

Special $\varepsilon$-uniformly convergent difference schemes for singularly perturbed boundary value problems for elliptic and parabolic equations are well developed, see, e.g., [1-9]. If the problem data are sufficiently smooth, for the parabolic equations with convection terms, then the order of $\varepsilon$-uniform convergence for the scheme studied in [9] is $\mathscr{O}\left(N^{-1} \ln N+N_{0}^{-1}\right)$, where $N$ and $N_{0}$ denote, respectively, the number of intervals in the space and time discretization. For this scheme the amount of computational work is primarily determined by the time discretization, which is of first order accuracy only. For reaction-diffusion problems in $[5,6]$ we have developed an algorithm based on the defect correction principle, which achieves a high order of time-accuracy and preserves $\varepsilon$-uniform accuracy in space.

To improve the effectivity of the algorithm, we also need efficient methods for solving the discretized problems. The paper [10] introduced parallel computational methods that allow us to accelerate the numerical solution of singularly perturbed boundary value problems for parabolic reaction-diffusion equations.

*This research was supported in part by the Netherlands Organization for Scientific Research NWO, dossiernr. 047.008.007, and by the Russian Foundation for Basic Research under Grant N 98-01-00362. 
Note that the method in [10] has only first order of accuracy with respect to the time variable. The direct use of the parallel method from [10] for the defect correction scheme does not allow to achieve the order of time accuracy higher than one.

In the present paper we develop new defect correction schemes for singularly perturbed convection-diffusion problems. In this way, we achieve a high order of accuracy for the time variable, maintaining $\varepsilon$-uniform convergence and first-order accuracy in space. For such schemes, as well as for the base schemes, we construct new schemes based on a domain decomposition method which allow both sequential and parallel computations. Thus, the present algorithm is a further efficient improvement over what was previously developed in [6] for the reactiondiffusion case. It should be noted that this parallel method is not iterative within a time step.

\section{Problem Formulation}

On the domain $\bar{G}$, where $G=(0,1) \times(0, T]$, with the boundary $S=\bar{G} \backslash G$ we consider the following singularly perturbed parabolic equation with Dirichlet boundary conditions:

$$
\begin{aligned}
L_{(2.1)} u(x, t) & \equiv\left\{\varepsilon a(x, t) \frac{\partial^{2}}{\partial x^{2}}+b(x, t) \frac{\partial}{\partial x}-c(x, t)-p(x, t) \frac{\partial}{\partial t}\right\} u(x, t) \\
& =f(x, t), \quad(x, t) \in G, \\
u(x, t) & =\varphi(x, t), \quad(x, t) \in S .
\end{aligned}
$$

For $S=S_{0} \cup S^{L}$, we distinguish the lateral boundary $S^{L}=\{(x, t): x=0$ or $x=1,0<t \leq T\}$, and the initial boundary $S_{0}=\{(x, t): x \in[0,1], t=0\}$. In (2.1) $a(x, t), b(x, t), c(x, t), p(x, t), f(x, t),(x, t) \in \bar{G}$, and $\varphi(x, t),(x, t) \in S$ are sufficiently smooth and bounded functions which satisfy

$$
0<a_{0} \leq a(x, t), 0<b_{0} \leq b(x, t), 0<p_{0} \leq p(x, t), c(x, t) \geq 0, \quad(x, t) \in \bar{G} .
$$

The real parameter $\varepsilon$ may take any small positive value, say $\varepsilon \in(0,1]$.

When the parameter $\varepsilon$ tends to zero in (2.1a), the solution exhibits a boundary layer in the neighbourhood of the set $S_{0}^{L}=\{(x, t): x=0,0 \leq t \leq T\}$, i.e., near the left side of the lateral boundary (or the outlow boundary). Such layers are described by an ordinary differential equation (regular boundary layers).

For problem (2.1) we construct a numerical method that has a higher order of accuracy with respect to the time and, in addition, allows for parallel solution of the difference equations. 


\section{Difference Scheme}

To solve problem (2.1) we first consider a classical finite difference method. On the set $\bar{G}$ we introduce the rectangular grid

$$
\bar{G}_{h}=\bar{\omega} \times \bar{\omega}_{0},
$$

where $\bar{\omega}$ is a (possibly) non-uniform grid of nodal points, $x^{i}$, in $[0,1], \bar{\omega}_{0}$ is a uniform grid on the interval $[0, T] ; N$ and $N_{0}$ are the numbers of intervals in the grids $\bar{\omega}$ and $\bar{\omega}_{0}$ respectively. We define $\tau=T / N_{0}, h^{i}=x^{i+1}-x^{i}, h=\max _{i} h^{i}$, $h \leq M / N$.

Here and below we denote by $M$ (or $m$ ) sufficiently large (or small) positive constants which do not depend on the value of parameter $\varepsilon$ or on the difference operators.

For problem (2.1) we use the difference scheme [11]

$$
\begin{gathered}
\Lambda_{(3.2)} z(x, t)=f(x, t), \quad(x, t) \in G_{h}, \\
z(x, t)=\varphi(x, t), \quad(x, t) \in S_{h} .
\end{gathered}
$$

Here $G_{h}=G \cap \bar{G}_{h}, S_{h}=S \cap \bar{G}_{h}, \Lambda_{(3.2)} \equiv \varepsilon a(x, t) \delta_{\bar{x} \hat{x}}+b(x, t) \delta_{x}-c(x, t)-p(x, t) \delta_{\bar{t}}$, $\delta_{x} z(x, t)$ is the forward difference operator, while $\delta_{\bar{x}} z(x, t)$ and $\delta_{\bar{t}} z(x, t)$ are the backward difference operators, and the difference derivative $\delta_{\bar{x} \hat{x}} z(x, t)$ is an approximation of the derivative $\frac{\partial^{2}}{\partial x^{2}} u(x, t)$ on the non-uniform mesh:

$$
\delta_{\bar{x} \hat{x}} z\left(x^{i}, t\right)=2\left(h^{i-1}+h^{i}\right)^{-1}\left[\delta_{x} z\left(x^{i}, t\right)-\delta_{\bar{x}} z\left(x^{i}, t\right)\right] .
$$

The difference scheme (3.2), (3.1) is monotone [11]. By the maximum principle and taking into account a-priori estimates of the derivatives (see Theorem 9.1 in the Appendix), we find the error estimate

$$
|u(x, t)-z(x, t)| \leq M\left(\varepsilon^{-2} N^{-1}+\tau\right), \quad(x, t) \in \bar{G}_{h} .
$$

The proof of (3.3) follows the lines of the classical convergence proof for monotone difference schemes $[11,9]$. This results in the following theorem.

Theorem 3.1. Let estimate (9.2), where $n=0$, hold for the solution of problem (2.1). Then, for a fixed value of the parameter $\varepsilon$, the solution of scheme (3.2), (3.1) converges to the solution of problem (2.1) with an error bound given by (3.3).

\section{The $\varepsilon$-Uniformly Convergent Method}

In this section we discuss an $\varepsilon$-uniformly convergent method for (2.1) by taking a special mesh, condensed in the neighbourhood of boundary layers. The location of the nodes is derived from a-priori estimates of the solution and its derivatives. The way to construct the mesh for problem (2.1) is the same as in $[5,7,12,13,9]$. More specifically, we take 


$$
\bar{G}_{h}^{*}=\bar{\omega}^{*}(\sigma) \times \bar{\omega}_{0},
$$

where $\bar{\omega}_{0}$ is the uniform mesh with step-size $\tau=T / N_{0}$, i.e. $\bar{\omega}_{0}=\bar{\omega}_{0(3.1)}$, and $\bar{\omega}^{*}=\bar{\omega}^{*}(\sigma)$ is a special piecewise uniform mesh depending on the parameter $\sigma \in \mathbb{R}$, which depends on $\varepsilon$ and $N$. We take $\sigma=\sigma_{(4.1)}(\varepsilon, N)=\min \left[1 / 2, m^{-1} \varepsilon \ln N\right]$, where $m=m_{(4.1)}$ is an arbitrary positive number from the interval $\left(0, m_{0}\right)$, $m_{0}=\min _{\bar{G}}\left[a^{-1}(x, t) b(x, t)\right]$. The mesh $\bar{\omega}^{*}(\sigma)$ is constructed as follows. The interval $[0,1]$ is divided in two parts $[0, \sigma],[\sigma, 1], 0<\sigma \leq 1 / 2$. In each part we use a uniform grid, with $N / 2$ subintervals in each interval $[0, \sigma]$ and $[\sigma, 1]$.

Theorem 4.1. If the solution of problem (2.1) satisfies the hypotheses of Theorem 9.1 (Appendix), where $n=0$, then the solution of scheme (3.2), (4.1) converges $\varepsilon$-uniformly to the solution of (2.1) and the following estimate holds:

$$
|u(x, t)-z(x, t)| \leq M\left(N^{-1} \ln N+\tau\right), \quad(x, t) \in \bar{G}_{h}^{*} .
$$

The proof of this theorem can be found in [9].

Remark 1. Under the conditions of Theorem 4.1, where $n=K \geq 0$, for the derivatives $\left(\partial^{k_{0}} / \partial t^{k_{0}}\right) u(x, t)$ and the divided differences $\delta_{l t} z(x, t)$, the following estimates hold:

$$
\begin{gathered}
\left|\frac{\partial^{k_{0}}}{\partial t^{k_{0}}} u(x, t)\right| \leq M_{(4.3)}^{\left(k_{0}\right)}, \quad(x, t) \in \bar{G}, k_{0} \leq K+2 ; \\
\left|\delta_{l \bar{t}} z(x, t)\right| \leq M_{(4.4)}^{(l)}, \quad(x, t) \in \bar{G}_{h(4.1)}, \quad t \geq l \tau, \quad l \leq K+1 .
\end{gathered}
$$

Here we denote by $\delta_{l t} z(x, t)$ the backward difference of order $l$ :

$$
\begin{gathered}
\delta_{l \bar{t}} z(x, t)=\left(\delta_{l-1 \bar{t}} z(x, t)-\delta_{l-1 \bar{t}} z(x, t-\tau)\right) / \tau, l \geq 1, \\
\delta_{0 \bar{t}} z(x, t)=z(x, t), \quad(x, t) \in \bar{G}_{h}, t \geq l \tau, l \geq 0 .
\end{gathered}
$$

\section{Schwarz Overlapping Method for Parabolic Equations}

We give the modified Schwarz method for boundary value problem (2.1), and for the solutions obtained we give the necessary and sufficient conditions of $\varepsilon$-uniform convergence.

5.1. We first describe Schwarz' classical method for problem (2.1). Let the set of open subdomains

$$
D^{k}, \quad k=1, \ldots, K
$$

with piecewise smooth boundaries $\Gamma^{k}, \Gamma^{k}=\Gamma\left(D^{k}\right)=\bar{D}^{k} \backslash D^{k}$, cover the domain $D$ : $D=\cup_{k=1}^{K} D^{k}$, and let 


$$
G^{k}=D^{k} \times(0, T], \quad k=1, \ldots, K .
$$

We denote by $D^{[k]}$ the union of the subdomains $D^{1}, \ldots, D^{K}$ which does not include the set $D^{k}$

$$
D^{[k]}=\bigcup_{i=1, i \neq k}^{K} D^{i}, \quad k=1, \ldots, K .
$$

Note that the sets $D^{k} \cap D^{[k]} \neq \emptyset$ for all $k=1, \ldots, K$. For simplicity, we assume that $D \backslash D^{[k]} \neq \emptyset, k=1, \ldots, K$. We denote the minimal overlap of the sets $D^{k}$ and $D^{[k]}$ by $\delta^{k}$ and $\delta$ denote the least value of $\delta^{k}, k=1, \ldots, K$, i.e.

$$
\min _{k, x^{1}, x^{2}} \rho\left(x^{1}, x^{2}\right)=\delta, \quad x^{1} \in \bar{D}^{k}, x^{2} \in \bar{D}^{[k]}, x^{1}, x^{2} \notin\left\{D^{k} \cap D^{[k]}\right\}, k=1, \ldots, K,
$$

where $\rho\left(x^{1}, x^{2}\right)$ is the distance between the points $x^{1}, x^{2} \in \bar{D}$. In general, the value $\delta$ may depend on the parameter $\varepsilon: \delta=\delta_{(5.2)}(\varepsilon)$.

Let

$$
u^{0}(x, t), \quad(x, t) \in \bar{G}
$$

be given an arbitrary function satisfying the condition (2.1b). We are to find the sequence of the functions $u^{r}(x, t),(x, t) \in \bar{G}, r=1,2, \ldots$ Let the function $u^{r}(x, t)$ be known. The function $u^{r+1}(x, t)$ is determined as follows. First we find the functions $u^{r+\frac{k}{K}}(x, t)$, that is the solution of the following problems

$$
\begin{gathered}
L_{(5.4)}\left(u^{r+\frac{k}{K}}(x, t)\right)=0, \quad(x, t) \in G^{k}, \\
u^{r+\frac{k}{K}}(x, t)=u^{r+\frac{k-1}{K}}(x, t), \quad(x, t) \in \bar{G} \backslash G^{k}, k=1, \ldots, K .
\end{gathered}
$$

The required function is defined by the relation

$$
u^{r+1}(x, t)=u^{r+\frac{K}{K}}(x, t), \quad r=0,1,2, \ldots
$$

In the case of boundary value problem (2.1) the operator $L_{(5.4)}$ in (5.3b) is defined

$$
L_{(5.4)}(u(x, t)) \equiv L_{(2.1)} u(x, t)-f(x, t), \quad(x, t) \in G .
$$

Each function $u^{r+\frac{k}{K}}(x, t),(x, t) \in \bar{G}$, is the solution of the Dirichlet problem on the set $\bar{G}^{k}$ and coincides with the function $u^{r+\frac{k-1}{K}}(x, t)$ on the set $\bar{G} \backslash G^{k}$. This process is a natural generalization of the classical Schwarz 'alternating' method.

In principle, we could give the conditions under which process (5.3), (5.4), (5.1) converges to the solution of boundary value problem (2.1) as $r \rightarrow \infty$, where $r$ is the number of iterations. However, in this paper we are interested in a noniterative variant solver based on the modified Schwarz method. 
5.2. Now we describe the modified Schwarz method. Let

be a uniform grid, just like $\bar{\omega}_{0(3.1)}$, on $[0, \mathrm{~T}]$ with stepsize $\tau$. By $G\left(t_{1}\right)$ we denote the strip

$$
G\left(t_{1}\right)=\left\{(x, t):(x, t) \in G, t_{1}<t \leq t_{1}+\tau\right\}, t_{1}, t_{1}+\tau \in \bar{\omega}_{0} .
$$

Let $S\left(t_{1}\right)=\bar{G}\left(t_{1}\right) \backslash G\left(t_{1}\right)$ be the boundary of $G\left(t_{1}\right)$ and let $v(x, t)=v\left(x, t ; t_{1}\right)$ be defined on $S\left(t_{1}\right)$. We denote an extension of the function $v(x, t)$ onto the whole set $\bar{G}\left(t_{1}\right)$ by $\bar{v}\left(x, t ; t_{1}\right)$. The function $\bar{v}\left(x, t ; t_{1}\right)$ is assumed to satisfy a Lipschitz condition with respect to $t$. We subdivide the strip $G\left(t_{1}\right)$ into sections $G^{k}\left(t_{1}\right)=$ $G^{k} \cap G\left(t_{1}\right), S^{k}\left(t_{1}\right)=\bar{G}^{k}\left(t_{1}\right) \backslash G^{k}\left(t_{1}\right)$.

Suppose the function $u(x, t),(x, t) \in \bar{G}$, for $t^{n} \in \bar{\omega}_{0}, t \leq t^{n}<T, n=0,1, \ldots$, $N_{0}-1$, has already been constructed. Now we construct the function $u(x, t)$ for $t \leq t^{n+1}$, i.e., we find the function $u(x, t)$ on the strip $G\left(t^{n}\right)$. This is done in the following way. First we find the functions $u^{k / K}(x, t)$ on the sections $\bar{G}^{k}\left(t^{n}\right)$, solving the boundary value problems

$$
\begin{aligned}
& L_{(5.4)}\left(u^{\frac{k}{K}}(x, t)\right)=0, \quad(x, t) \in G^{k}\left(t^{n}\right), \\
& \left.u^{\frac{k}{K}}(x, t)=\left\{\begin{array}{cc}
\bar{u}\left(x, t ; t^{n}\right), & k=1, \\
u^{\frac{k-1}{K}}(x, t), & k \geq 2
\end{array}\right\}, \quad(x, t) \in S^{k}\left(t^{n}\right)\right\} \quad \text { for }(x, t) \in \bar{G}^{k}\left(t^{n}\right), \\
& k=1, \ldots, K ; t^{n} \in \bar{\omega}_{0}, n \leq N_{0}-1 \text {. }
\end{aligned}
$$

Here having $u^{k} / K(x, t)$ on $\bar{G}^{k}\left(t^{n}\right)$, we extend these functions for each value $k$ onto the whole strip $\bar{G}\left(t^{n}\right)$ in the following way

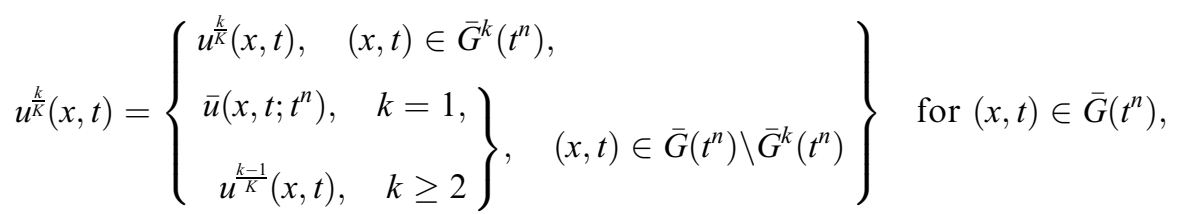

$$
\begin{aligned}
& k=1, \ldots, K, t^{n} \in \bar{\omega}_{0} \text {. }
\end{aligned}
$$

Having $u^{k / K}(x, t)$, for $k=K$ we define the function $u(x, t)$ on the whole strip $\bar{G}\left(t^{n}\right)$ by

$$
u(x, t)=u^{\frac{K}{K}}(x, t), \quad(x, t) \in \bar{G}\left(t^{n}\right), t^{n} \in \bar{\omega}_{0} .
$$

Thereby we have the function $u(x, t)$ on the domain $\bar{G}$ for $t \in\left[0, t^{n+1}\right]$.

In the relations $(5.5 \mathrm{~b}),(5.5 \mathrm{c})$ the function $\bar{u}\left(x, t ; t^{n}\right)$ is constructed on the base of the function $\bar{v}\left(x, t ; t^{n}\right)$ 


$$
\bar{u}\left(x, t ; t^{n}\right)=\bar{v}\left(x, t ; t^{n}\right), \quad(x, t) \in \bar{G}\left(t^{n}\right) .
$$

Using $v\left(x, t ; t^{n}\right)$ which is defined on the boundary $S\left(t^{n}\right)$ in $(5.5 \mathrm{~g})$, we find the function

$$
\begin{gathered}
\bar{v}\left(x, t ; t^{n}\right), \quad(x, t) \in \bar{G}\left(t^{n}\right), \\
\text { supposing } \bar{v}\left(x, t ; t^{n}\right)=v\left(x, t ; t^{n}\right) \quad \text { for }(x, t) \in S\left(t^{n}\right) \\
\text { and } \bar{v}\left(x, t ; t^{n}\right)=v\left(x, t^{n} ; t^{n}\right) \quad \text { for }(x, t) \in G\left(t^{n}\right) .
\end{gathered}
$$

Here

$$
v\left(x, t ; t^{n}\right)= \begin{cases}\varphi(x, t), \quad(x, t) \in S\left(t^{n}\right), & t^{n}=t^{0}=0, \\ \varphi(x, t), & (x, t) \in S\left(t^{n}\right) \cap S, t \geq t^{n}, \\ u(x, t), & (x, t) \in S\left(t^{n}\right) \backslash S, t=t^{n} \\ n=0,1, \ldots, N_{0}-1 . & t^{n}>0,(x, t) \in S\left(t^{n}\right),\end{cases}
$$

Thus, the function $\bar{u}\left(x, t ; t^{n}\right)$ on $\bar{G}\left(t^{n}\right)$ has been constructed.

The function $u^{\frac{k}{k}}(x, t)$ on each strip $\bar{G}\left(t^{n}\right)$ is the solution of the Dirichlet problem on the section $\bar{G}^{k}\left(t^{n}\right)$, whereas on the set $\bar{G}\left(t^{n}\right) \backslash G^{k}\left(t^{n}\right)$ it coincides with the function $\bar{u}\left(x, t ; t^{n}\right)(x, t) \in \bar{G}\left(t^{n}\right)$ for $k=1$, and with the function $u^{\frac{k-1}{K}}(x, t),(x, t) \in \bar{G}\left(t^{n}\right)$ for $k \geq 2$. We thus find the function $u(x, t),(x, t) \in \bar{G}$, the solution of process (5.5), (5.4), (5.1), which we call the modified Schwarz method.

Note that the modified Schwarz method is not an iterative process in the strict sense. The boundary value problems in (5.5), (5.4), (5.1) are solved only once at those points of $\bar{G}$ which do not belong to the intersection of the subdomains. The boundary value problem is solved twice only on the intersection of the subdomains.

In the continuous domain decomposition method (5.5), (5.4), (5.1) the intermediate problems on the subsets $\bar{D}_{(5.1)}^{k}, k=1, \ldots, K$ are solved sequentially.

Using the comparison theorems $[14,15]$, we come to the estimate

$$
\left|u(x, t)-u_{(5.5)}(x, t)\right| \leq Q(\varepsilon, \delta) N_{0}^{-1}, \quad(x, t) \in \bar{G},
$$

where $u_{(5.5)}(x, t)$ is the solution of the process (5.5), (5.4), (5.1), $\delta=\delta_{(5.2)}(\varepsilon)$, i.e., the function $u_{(5.5)}(x, t)$ converges, as $N_{0} \rightarrow \infty$, to the solution of boundary value problem (2.1) for each fixed value of the parameter $\varepsilon$. Note that, generally speaking, the function $u_{(5.5)}(x, t)$ for $\delta=0$ does not converge to the solution of boundary value problem (2.1) as $N_{0} \rightarrow \infty$. Under the condition (see [16], Chapter 10 of [7]) 


$$
\delta=\delta_{(5.2)}(\varepsilon)>0, \quad \varepsilon \in(0,1], \inf _{\varepsilon \in(0,1]}\left[\varepsilon^{-1} \delta_{(5.2)}(\varepsilon)\right]>0
$$

which is equivalent to the condition $\delta=\delta_{(5.2)}(\varepsilon) \geq m_{(5.6)} \varepsilon, \varepsilon \in(0,1]$, the function $u_{(5.5)}(x, t)$ converges $\varepsilon$-uniformly as $N_{0} \rightarrow \infty$ :

$$
\left|u(x, t)-u_{(5.5)}(x, t)\right| \leq M N_{0}^{-1}, \quad(x, t) \in \bar{G} .
$$

If condition (5.6) is violated and the value $\delta$ satisfies the condition

$$
\delta=\delta_{(5.2)}(\varepsilon)>0, \quad \varepsilon \in(0,1], \inf _{\varepsilon \in(0,1]}\left[\varepsilon^{-1} \delta_{(5.2)}(\varepsilon)\right]=0,
$$

the function $u_{(5.5)}(x, t)$ does not converge $\varepsilon$-uniformly.

5.3. Here we describe the continuous variant of the modified Schwarz method that admits parallel computations on $P \geq 1$ processors.

Let $D^{k}, k=1, \ldots, K$ be the subdomains from (5.1a) and let each $D^{k}$ be partioned in $P$ disjoint (possibly empty) parts

$$
D^{k}=\bigcup_{p=1}^{P} D_{p}^{k}, \quad k=1, \ldots, K
$$

where $\bar{D}_{i}^{k} \cap \bar{D}_{j}^{k}=\emptyset, i \neq j$. We set

$$
G_{p}^{k}=D_{p}^{k} \times(0, T], \quad p=1, \ldots, P, k=1, \ldots, K .
$$

We find the function $u(x, t)$ by solving problems (5.9) similar to (5.5), but now on the set $\bar{G}_{p}^{k}\left(t^{n}\right)$ instead of $\bar{G}^{k}\left(t^{n}\right)$

$$
\begin{aligned}
& L_{(5.4)}\left(u_{p}^{\frac{k}{K}}(x, t)=0, \quad(x, t) \in G_{p}^{k}\left(t^{n}\right),\right. \\
& u_{p}^{\frac{k}{K}}(x, t)=\left\{\begin{array}{cc}
\bar{u}\left(x, t ; t^{n}\right), & k=1, \\
u^{\frac{k-1}{K}}(x, t), & k \geq 2
\end{array}\right\}, \quad(x, t) \in S_{p}^{k}\left(t^{n}\right), p=1, \ldots, P \\
& \text { for }(x, t) \in \bar{G}_{p}^{k}\left(t^{n}\right), k=1, \ldots, K, t^{n} \in \bar{\omega}_{0}, n \leq N_{0}-1 \text {; } \\
& \left.u^{\frac{k}{K}}(x, t)=\left\{\begin{array}{ll}
u_{p}^{\frac{k}{K}}(x, t), & (x, t) \in \bar{G}_{p}^{k}\left(t^{n}\right), p=1, \ldots, P, \\
\bar{u}\left(x, t ; t^{n}\right), & k=1, \\
u^{\frac{k-1}{K}}(x, t), & k \geq 2
\end{array}\right\}, \quad(x, t) \in \bar{G}\left(t^{n}\right) \backslash \bigcup_{p=1}^{P} \bar{G}_{p}^{k}\left(t^{n}\right)\right\} \\
& \text { for }(x, t) \in \bar{G}\left(t^{n}\right), k=1, \ldots, K, t^{n} \in \bar{\omega}_{0} \text {; } \\
& u(x, t)=u^{\frac{K}{K}}(x, t), \quad(x, t) \in \bar{G}\left(t^{n}\right), t^{n} \in \bar{\omega}_{0} .
\end{aligned}
$$


The function $\bar{u}\left(x, t ; t^{n}\right)=\bar{v}\left(x, t ; t^{n}\right),(x, t) \in \bar{G}\left(t^{n}\right), \quad t^{n} \in \bar{\omega}_{0} . \quad$ The function $\bar{v}\left(x, t ; t^{n}\right), \quad(x, t) \in \bar{G}\left(t^{n}\right)$ is determined like that in (5.5f).

Stepwise, for $n=1,2, \ldots$, we find the function $u_{(5.9)}(x, t),(x, t) \in \bar{G}$, i.e., the solution of process (5.9), (5.8). We call this the modified continuous Schwarz method for $P$ "processors".

The scheme (5.9) on the decomposition (5.8) can be written in the "operator" form

$$
Q\left(u(x, t) ; \omega_{0}, f(\cdot), \varphi(\cdot), \psi(\cdot)\right)=0, \quad(x, t) \in G .
$$

Here the function $\psi\left(x, t ; t^{n}\right),(x, t) \in G\left(t^{n}\right)$ defines the prolonged function $\bar{u}\left(x, t ; t^{n}\right)$ :

$$
\bar{u}\left(x, t ; t^{n}\right)=\left\{\begin{array}{l}
v\left(x, t ; t^{n}\right), \quad(x, t) \in S\left(t^{n}\right), \\
v\left(x, t^{n} ; t^{n}\right)+\psi\left(x, t ; t^{n}\right), \quad(x, t) \in G\left(t^{n}\right)
\end{array}\right\}, \quad(x, t) \in \bar{G}\left(t^{n}\right)
$$

so that in the case of the conditions (5.5e), (5.5f), simply, $\psi\left(x, t ; t^{n}\right) \equiv 0$. The problem (5.9), (5.8) for $P=1$ is identical with problem (5.5), (5.1).

In the continuous domain decomposition method (5.9), (5.8) the intermediate problems on the subsets $\bar{D}_{p(5.8)}^{k}, p=1, \ldots, P, k=1, \ldots, K$ can be solved independently of each other, for all $p=1, \ldots, P$. For this construction the following theorem [10] is useful.

Theorem 5.1. The condition (5.6) is necessary and sufficient for the E-uniform convergence (as $\left.N_{0} \rightarrow \infty\right)$ of $u_{(5.9 b)}(x, t)$ i.e., the solution of process (5.9), (5.8) with $P \geq 1$, to $u(x, t)$, i.e., the solution of boundary value problem (2.1).

\section{Difference Schemes Based on the Schwarz Method}

6.1. Here we construct a difference scheme based on the process (5.5), (5.1) and give the necessary and sufficient conditions for $\varepsilon$-uniform convergence of this scheme. We introduce the rectangular grids on each set $\bar{G}^{k}$ and $\bar{G}_{p}^{k}$ :

$$
\bar{G}_{h}^{k}=\bar{G}^{k} \cap \bar{G}_{h(3.1)}, \quad \bar{G}_{p h}^{k}=\bar{G}_{p}^{k} \cap \bar{G}_{h(3.1)},
$$

or

$$
\bar{G}_{h}^{k *}=\bar{G}^{k} \cap \bar{G}_{h(4.1)}^{*}, \quad \bar{G}_{p h}^{k *}=\bar{G}_{p}^{k} \cap \bar{G}_{h(4.1)}^{*} .
$$

where $\bar{G}_{p h}^{k}=\bar{G}_{p, h}^{k}$. We assume that the boundaries of $\bar{G}^{k}$ and $\bar{G}_{p}^{k}$ pass through the nodes of the grid $\bar{G}_{h}$ and $\bar{G}_{h}^{*}$ respectively.

Now we introduce the discrete function $v(x, t)=v\left(x, t ; t_{1}\right)$ defined on the boundary of the discrete strip $S_{h}\left(t_{1}\right)=S\left(t_{1}\right) \cap \bar{G}_{h}, t_{1} \in \bar{\omega}_{0}$. By $\bar{v}\left(x, t ; t_{1}\right)$ we denote the extension of this function $v(x, t)$ to the discrete set $\bar{G}_{h}\left(t_{1}\right)=\bar{G}\left(t_{1}\right) \cap \bar{G}_{h}$. The function 
$\bar{v}\left(x, t ; t_{1}\right)$ is considered to satisfy the Lipschitz condition with respect to $t$. The "strip" $\bar{G}_{h}\left(t_{1}\right)$ consists only of two time levels

$$
\bar{G}_{h}\left(t_{1}\right)=\left\{\bar{\omega} \times\left[t=t_{1}\right]\right\} \cup\left\{\bar{\omega} \times\left[t=t_{1}+\tau\right]\right\},
$$

where $\bar{\omega}$ was introduced in (3.1).

Now we find the discrete solutions $z^{\frac{k}{K}}(x, t)$ by a procedure similar to (5.5). Assuming that $z(x, t), t \leq t^{n}$, has been computed, we solve on the strip $\bar{G}_{h}\left(t^{n}\right)$ the problems

$$
\begin{aligned}
& \Lambda_{(6.3)}\left(z^{\frac{k}{K}}(x, t)\right)=0, \quad(x, t) \in G_{h}^{k}\left(t^{n}\right), \\
& \left.z^{\frac{k}{K}}(x, t)=\left\{\begin{array}{cc}
\bar{z}\left(x, t ; t^{n}\right), & k=1, \\
z^{\frac{k-1}{K}}(x, t), & k \geq 2
\end{array}\right\}, \quad(x, t) \in S_{h}^{k}\left(t^{n}\right)\right\} \quad \text { for }(x, t) \in \bar{G}_{h}^{k}\left(t^{n}\right), \\
& k=1, \ldots, K, t^{n} \in \bar{\omega}_{0}, n \leq N_{0}-1 \text {; }
\end{aligned}
$$

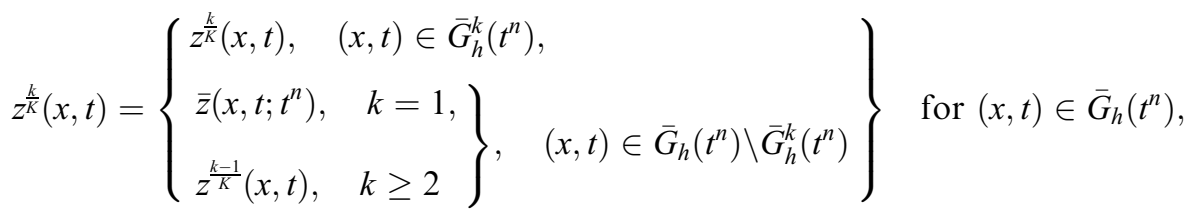

$$
\begin{aligned}
& k=1, \ldots, K, t^{n} \in \bar{\omega}_{0} \text {. }
\end{aligned}
$$

The required function $z(x, t)$ on the strip $\bar{G}_{h}\left(t^{n}\right)$ is defined by the relation

$$
z(x, t)=z^{\frac{K}{K}}(x, t), \quad(x, t) \in \bar{G}_{h}\left(t^{n}\right), t^{n} \in \bar{\omega}_{0} .
$$

In the relations $(6.3 \mathrm{a}),(6.3 \mathrm{~b})$

$$
\bar{z}\left(x, t ; t^{n}\right)=\bar{v}\left(x, t ; t^{n}\right), \quad(x, t) \in \bar{G}_{h}\left(t^{n}\right), t^{n} \in \bar{\omega}_{0} .
$$

The function $\bar{v}\left(x, t ; t^{n}\right),(x, t) \in \bar{G}_{h}\left(t^{n}\right)$ is found from $v\left(x, t ; t^{n}\right),(x, t) \in S_{h}\left(t^{n}\right)$,

$$
\bar{v}\left(x, t ; t^{n}\right)=\left\{\begin{array}{lc}
v\left(x, t ; t^{n}\right), & (x, t) \in S_{h}\left(t^{n}\right), \\
v\left(x, t^{n} ; t^{n}\right), & (x, t) \in G_{h}\left(t^{n}\right)
\end{array}\right\}, \quad(x, t) \in \bar{G}_{h}\left(t^{n}\right)
$$

where

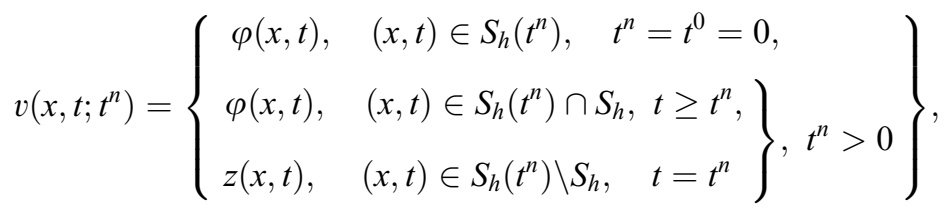

$$
\begin{aligned}
& (x, t) \in S_{h}\left(t^{n}\right), n=0,1, \ldots, N_{0}-1 \text {. }
\end{aligned}
$$


On each strip $\bar{G}_{h}\left(t^{n}\right)$ the function $z^{\frac{k}{K}}(x, t)$ is the solution of the discrete Dirichlet problem on the set $\bar{G}_{h}^{k}\left(t^{n}\right)$. On the remaining part $\bar{G}_{h}\left(t^{n}\right) \backslash G_{h}^{k}\left(t^{n}\right)$, it coincides for $k=1$ with the function $\bar{z}\left(x, t ; t^{n}\right),(x, t) \in \bar{G}_{h}\left(t^{n}\right)$ and for $k \geq 2$ with the function $z^{\frac{k-1}{K}}(x, t),(x, t) \in \bar{G}_{h}\left(t^{n}\right)$. We define the operator $\Lambda_{(6.3)}$ by the relation

$$
\Lambda_{(6.3)}(z(x, t)) \equiv \Lambda_{(3.2)} z(x, t)-f(x, t), \quad(x, t) \in G_{h} .
$$

It is required to find the function $z_{(6.3)}(x, t),(x, t) \in \bar{G}_{h}$, i.e., the solution of difference scheme (6.3) either on the mesh (4.1) or on the mesh (3.1). The difference scheme (6.3) can symbolically be written in the operator form

$$
Q_{(6.3)}\left(z_{(6.3)}(x, t) ; f(\cdot), \varphi(\cdot), \psi(\cdot)\right)=0, \quad(x, t) \in \bar{G}_{h} .
$$

Similar to (5.9e), here the function $\psi\left(x, t ; t^{n}\right),(x, t) \in G_{h}\left(t^{n}\right)$ defines the function $\bar{z}\left(x, t ; t^{n}\right)$ :

$$
\bar{z}\left(x, t ; t^{n}\right)=\left\{\begin{array}{ll}
v\left(x, t ; t^{n}\right), & (x, t) \in S_{h}\left(t^{n}\right), \\
v\left(x, t^{n} ; t^{n}\right)+\psi\left(x, t ; t^{n}\right), & (x, t) \in G_{h}\left(t^{n}\right)
\end{array}\right\},(x, t) \in \bar{G}_{h}\left(t^{n}\right) .
$$

In the above case of conditions (6.3d), (6.3e) we have $\psi\left(x, t ; t^{n}\right) \equiv 0$.

In the discrete domain decomposition method (6.3), the intermediate problems on the subsets $\bar{D}_{h}^{k}=\bar{D}_{(5.1)}^{k} \cap \bar{D}_{h}$ are solved sequentially. Thus, to solve boundary value problem (2.1), here we used difference scheme (6.3), (3.1), which is the discrete equivalent of (5.5), (5.1). In the following section we extend this to the "parallel" case (5.9).

6.2. To describe the difference scheme that approximates process (5.9), (5.8) with $P$ parallel processes, assume that $z(x, t)$ is known for $t \leq t^{n}$, then we solve the problems

$$
\begin{aligned}
& \Lambda_{(6.3)}\left(z_{p}^{\frac{k}{K}}(x, t)\right)=0, \quad(x, t) \in G_{p h}^{k}\left(t^{n}\right), \\
& z_{p}^{\frac{k}{K}}(x, t)=\left\{\begin{array}{cc}
\bar{z}\left(x, t ; t^{n}\right), & k=1, \\
z^{\frac{k-1}{K}}(x, t), & k \geq 2
\end{array}\right\}, \quad(x, t) \in S_{p h}^{k}\left(t^{n}\right), p=1, \ldots, P \\
& \text { for }(x, t) \in \bar{G}_{p h}^{k}\left(t^{n}\right), k=1, \ldots, K, t^{n} \in \bar{\omega}_{0}, n \leq N_{0}-1 \text {; }
\end{aligned}
$$

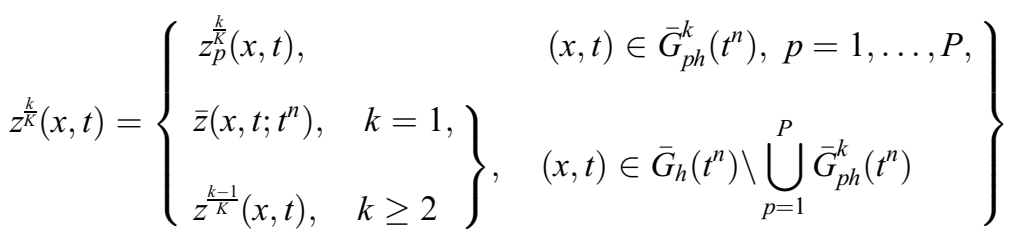

$$
\begin{aligned}
& \text { for }(x, t) \in \bar{G}_{h}\left(t^{n}\right), k=1, \ldots, K, t^{n} \in \bar{\omega}_{0} \text {. }
\end{aligned}
$$


We define the function $z_{(6.5)}(x, t)$ on the strip $\bar{G}_{h}\left(t^{n}\right)$ by the relation

$$
z_{(6.5)}(x, t)=z^{\frac{K}{K}}(x, t),(x, t) \in \bar{G}_{h}\left(t^{n}\right), t^{n} \in \bar{\omega}_{0} .
$$

In (6.5a) $\bar{z}\left(x, t ; t^{n}\right)=\bar{v}\left(x, t ; t^{n}\right),(x, t) \in \bar{G}_{h}\left(t^{n}\right)$. The function $\bar{v}\left(x, t ; t^{n}\right),(x, t) \in \bar{G}_{h}\left(t^{n}\right)$ can be found from $v\left(x, t ; t^{n}\right),(x, t) \in S_{h}\left(t^{n}\right)$, which is determined by relation (6.3e).

The difference scheme (6.5) can be written in the operator form

$$
Q_{(6.5)}\left(z_{(6.5)}(x, t) ; f(\cdot), \varphi(\cdot), \psi(\cdot)\right)=0, \quad(x, t) \in \bar{G}_{h},
$$

with $\psi\left(x, t ; t^{n}\right) \equiv 0$.

In the discrete domain decomposition method (6.5), (3.1) the intermediate problems on the subsets $\bar{D}_{p h}^{k}=\bar{D}_{p(5.8)}^{k} \cap \bar{D}_{h}$ are solved independent of each other ("in parallel") for all $p=1, \ldots, P$. For $P=1$ the difference scheme (6.5), (3.1) turns into (6.3), (3.1).

Under condition (5.6), using a standard technique of the comparison theorems (see, e.g., $[11,9]$ ), we get the estimate

$$
\left|z_{(3.2)}(x, t)-z_{(6.5)}(x, t)\right| \leq M N_{0}^{-1}, \quad(x, t) \in \bar{G}_{h},
$$

where $z_{(3.2)}(x, t)$ and $z_{(6.5)}(x, t)$ are the solutions of the difference schemes (3.2), (3.1) and (6.5), (3.1), respectively.

6.3. A technique similar to the one explained in $[5,6]$ gives us errors bounds for the discrete solutions that are obtained by the difference schemes described above. Under condition (5.6), if we use the difference schemes (6.5), (3.1) and (6.5), (4.1), we obtain the following error estimates

$$
\begin{aligned}
& \left|u(x, t)-z_{(6.5)}(x, t)\right| \leq M\left(\varepsilon^{-2} N^{-1}+\tau\right), \quad(x, t) \in \bar{G}_{h(3.1)}, \\
& \left|u(x, t)-z_{(6.5)}(x, t)\right| \leq M\left(N^{-1} \ln N+\tau\right), \quad(x, t) \in \bar{G}_{h(4.1)}^{*}
\end{aligned}
$$

The above formulation allows us to briefly summarize a result obtained in [10].

Theorem 6.1. Let the hypotheses of Theorem 4.1 hold for the data of boundary value problem (2.1) and its solution. Then, under condition (5.6) and for $N, N_{0} \rightarrow \infty$, the solution of the difference scheme (6.5), (4.1) (or scheme (6.5), (3.1)) converges to the solution of (2.1) E-uniformly (for a fixed value of $\varepsilon$ ). The estimates (6.6), (6.7) hold for the solutions of these difference schemes.

Remark 1. If the condition $n=0$ in Theorem 4.1 is replaced by $n=K, K \geq-1$, the following estimate holds

$$
\left|\delta_{l \bar{t}}\left(z_{(3.2)}(x, t)-z_{(6.5)}(x, t)\right)\right| \leq M M_{(4.3)}^{(l+1)} N_{0}^{-1}, \quad(x, t) \in \bar{G}_{h}, t \geq l \tau, l \leq K+1 .
$$


Remark 2. Note that the estimates (6.7a) and (6.7b) are similar to the estimates (3.3) and (4.2). It means that the domain decomposition preserves the accuracy of the base schemes.

\section{Improved Time-Accuracy}

\subsection{A Scheme Based on Defect Correction}

The technique used in this paper to improve time-accuracy is based on the one in [5]. For the difference scheme (3.2), (4.1) the error in the approximation of the partial derivative $(\partial / \partial t) u(x, t)$ is caused by the divided difference $\delta_{\bar{t}} z(x, t)$ and is associated with the truncation error given by the relation

$$
\frac{\partial}{\partial t} u(x, t)-\delta_{t} u(x, t)=2^{-1} \tau \frac{\partial^{2}}{\partial t^{2}} u(x, t)-6^{-1} \tau^{2} \frac{\partial^{3}}{\partial t^{3}} u(x, t-\vartheta),
$$

where $\vartheta \in[0, \tau]$. Therefore we now use for the approximation of $(\partial / \partial t) u(x, t)$ the expression $\delta_{\bar{t}} u(x, t)+\tau \delta_{\bar{t} \bar{t}} u(x, t) / 2$, where $\delta_{\bar{t} \bar{t}} u(x, t) \equiv \delta_{t \bar{t}} u(x, t-\tau), \delta_{t \bar{t}} u(x, t)$ is the second central divided difference. We can evaluate a better approximation than (3.2a) by defect correction

$$
\Lambda_{(3.2)} z^{c}(x, t)=f(x, t)+2^{-1} p(x, t) \tau \frac{\partial^{2}}{\partial t^{2}} u(x, t),
$$

with $x \in \bar{\omega}$ and $t \in \bar{\omega}_{0}$, where $\bar{\omega}$ and $\bar{\omega}_{0}$ are as in $(3.1) ; \tau$ is step-size of the grid $\bar{\omega}_{0}$; $z^{c}(x, t)$ is the "corrected" solution. Instead of $\left(\partial^{2} / \partial t^{2}\right) u(x, t)$ we shall use $\delta_{\bar{t} \bar{t}} z(x, t)$, where $z(x, t),(x, t) \in G_{h(4.1)}$ is the solution of the difference scheme (3.2), (4.1). The new solution $z^{c}(x, t)$ has a consistency error $\mathcal{O}\left(\tau^{2}\right)$.

\subsection{The Defect Correction Schemes of Second-Order Accuracy in Time}

Constructing the difference scheme in (7.2), instead of $\left(\partial^{2} / \partial t^{2}\right) u(x, t)$ we use $\delta_{2 \bar{t}} z(x, t)$, the second divided difference of the solution to the discrete problem (3.2), (4.1). On $\bar{G}_{h}$ we write the finite difference scheme (3.2) as

$$
\begin{array}{rlrl}
\Lambda_{(3.2)} z^{(1)}(x, t) & =f(x, t), & & (x, t) \in G_{h}, \\
z^{(1)}(x, t) & =\varphi(x, t), & (x, t) \in S_{h},
\end{array}
$$

where $z^{(1)}(x, t)$ is the uncorrected solution. For the corrected solution $z^{(2)}(x, t)$ we solve the problem for $(x, t) \in G_{h}$

$$
\begin{aligned}
\Lambda_{(3.2)} z^{(2)}(x, t) & =f(x, t)+\left\{\begin{array}{ll}
p(x, t) 2^{-1} \tau \frac{\partial^{2}}{\partial t^{2}} u(x, 0), & t=\tau, \\
p(x, t) 2^{-1} \tau \delta_{2 \bar{t}} z^{(1)}(x, t), & t \geq 2 \tau
\end{array}\right\}, \quad(x, t) \in G_{h}, \\
z^{(2)}(x, t) & =\varphi(x, t), \quad(x, t) \in S_{h} .
\end{aligned}
$$

Here the derivative $\frac{\partial^{2}}{\partial t^{2}} u(x, 0)$ is obtained from (2.1a). 
For simplicity, in the remainder of this section we consider a homogeneous initial condition:

$$
\varphi(x, 0)=0, \quad x \in \bar{D} .
$$

Under this condition, the following estimate similar to [6] holds for the solution of problem (7.4), (4.1):

$$
\left|u(x, t)-z^{(2)}(x, t)\right| \leq M\left[N^{-1} \ln N+\tau^{2}\right], \quad(x, t) \in \bar{G}_{h} .
$$

Theorem 7.1. Let condition (7.5) hold and assume in (2.1) that $a, b, c, p$, $f \in H^{(\alpha+2 n-2)}(\bar{G}), \varphi \in H^{(\alpha+2 n)}(\bar{G}), \alpha>4, n=K, K \geq 1$, and let condition (9.3) and estimates (9.5), (9.6) be satisfied for $n=K$. Then for the solution of difference scheme (7.4), (4.1) the estimate (7.6) holds.

\subsection{The Defect Correction Schemes of Third-Order Accuracy in Time}

The above procedure can be used to obtain an arbitrarily large order of accuracy in time. Here we only show how to construct the difference scheme of third order accuracy. On the grid $\bar{G}_{h}$ we consider the difference scheme

$$
\begin{aligned}
& \Lambda_{(3.2) z^{(3)}(x, t)=f(x, t)} \\
& \quad+\left\{\begin{array}{l}
p(x, t)\left(C_{11} \tau \frac{\partial^{2}}{\partial t^{2}} u(x, 0)+C_{12} \tau^{2} \frac{\partial^{3}}{\partial t^{3}} u(x, 0)\right), \quad t=\tau, \\
p(x, t)\left(C_{21} \tau \frac{\partial^{2}}{\partial t^{2}} u(x, 0)+C_{22} \tau^{2} \frac{\partial^{3}}{\partial t^{3}} u(x, 0)\right), \quad t=2 \tau, \quad(x, t) \in G_{h}, \\
p(x, t)\left(C_{31} \tau \delta_{2 t^{2}} z^{(2)}(x, t)+C_{32} \tau^{2} \delta_{3 t^{2}} z^{(1)}(x, t)\right), \quad t \geq 3 \tau
\end{array}\right\}, \quad(x, t) \in S_{h} .
\end{aligned}
$$

Here $z^{(1)}(x, t)$ and $z^{(2)}(x, t)$ are the solutions of problems (7.3), (4.1) and (7.4), (4.1) respectively, the derivatives $\left(\partial^{2} / \partial t^{2}\right) u(x, 0),\left(\partial^{3} / \partial t^{3}\right) u(x, 0)$ are again obtained from (2.1a), the coefficients $C_{i j}$ are determined below. They are chosen to satisfy the following conditions:

$$
\begin{aligned}
\frac{\partial}{\partial t} u(x, t) & =\delta_{\bar{t}} u(x, t)+C_{11} \tau \frac{\partial^{2}}{\partial t^{2}} u(x, t-\tau)+C_{12} \tau^{2} \frac{\partial^{3}}{\partial t^{3}} u(x, t-\tau)+\mathcal{O}\left(\tau^{3}\right), \\
\frac{\partial}{\partial t} u(x, t) & =\delta_{\bar{t}} u(x, t)+C_{21} \tau \frac{\partial^{2}}{\partial t^{2}} u(x, t-2 \tau)+C_{22} \tau^{2} \frac{\partial^{3}}{\partial t^{3}} u(x, t-2 \tau)+\mathcal{O}\left(\tau^{3}\right), \\
\frac{\partial}{\partial t} u(x, t) & =\delta_{\bar{t}} u(x, t)+C_{31} \tau \delta_{2 \bar{t}} u(x, t)+C_{32} \tau^{2} \delta_{3 \bar{t}} u(x, t)+\mathcal{O}\left(\tau^{3}\right) .
\end{aligned}
$$


It follows that

$$
C_{11}=C_{21}=C_{31}=1 / 2, C_{12}=C_{32}=1 / 3, C_{22}=5 / 6 .
$$

By $z^{(3)}(x, t)$ we denote the solution of the difference scheme (7.7), and again, for simplicity, we assume the homogeneous initial condition

$$
\varphi(x, 0)=0, \quad f(x, 0)=0, \quad x \in \bar{D} .
$$

Similarly to [6] we obtain, under condition (7.8), the following error bound for the solution of (7.7):

$$
\left|u(x, t)-z^{(3)}(x, t)\right| \leq M\left[N^{-1} \ln N+\tau^{3}\right], \quad(x, t) \in \bar{G}_{h} .
$$

This is more precisely formulated in the following theorem.

Theorem 7.2. Let conditions (7.8) hold and assume in (2.1) that $a, b, c, p$, $f \in H^{(\alpha+2 n-2)}(\bar{G}), \varphi \in H^{(\alpha+2 n)}(\bar{G}), \alpha>4, n=K, K \geq 2$ and let condition (9.3) and estimates (9.5), (9.6) be satisfied for $n=K$. Then for the solution of scheme (7.7), (4.1) the estimate (7.9) is valid.

Illustrative numerical results without domain decomposition are discussed in [6]. These results demonstrate the efficiency of the defect correction technique in improving the accuracy with respect to the time variable. However, in this paper we are interested in distributing the above algorithm over a number of independent ("parallel") processes.

\section{Parallel Method Based on Defect Correction}

\subsection{The Difference Schemes of Second-Order Accuracy in $\tau$}

8.1.1. Now we describe a finite difference scheme (6.3) constructed for the modified Schwarz method (5.5) with $P=1$ in the case of defect correction. To approximate the alternating process (5.5), we apply the defect correction scheme (7.4), (4.1) to the discrete equations (6.3), (6.2).

First we find the function $z^{(1)}(x, t),(x, t) \in \bar{G}_{h}\left(t^{n}\right)$, solving problem (6.3), (6.2)

$$
z^{(1)}(x, t) \equiv z_{(6.3 ; 6.2)}(x, t)
$$

where $z^{\frac{k}{K}}(x, t)$ and $z(x, t)$ are now denoted by $z^{(1) \frac{k}{K}}(x, t)$ and $z^{(1)}(x, t)$, respectively. For precise reference in the sequel, we rewrite the procedure (6.3), (6.2) as

$$
\begin{aligned}
& \Lambda_{(6.3 ; 6.2)}\left(z^{(1) \frac{k}{K}}(x, t)\right)=0, \quad(x, t) \in G_{h}^{k}\left(t^{n}\right), \\
& z^{(1) \frac{k}{K}}(x, t)=\left\{\begin{array}{ll}
\bar{z}^{(1)}\left(x, t ; t^{n}\right), & k=1, \\
z^{(1) \frac{k-1}{K}}(x, t), & k \geq 2
\end{array}\right\}, \quad(x, t) \in S_{h}^{k}\left(t^{n}\right) \\
& \text { for }(x, t) \in \bar{G}_{h}^{k}\left(t^{n}\right), k=1, \ldots, K, t^{n} \in \bar{\omega}_{0} \text {; }
\end{aligned}
$$




$$
\begin{aligned}
& \left.z^{(1) \frac{k}{K}}(x, t)=\left\{\begin{array}{ll}
z^{(1) \frac{k}{K}}(x, t), & (x, t) \in \bar{G}_{h}^{k}\left(t^{n}\right), \\
\bar{z}^{(1)}\left(x, t ; t^{n}\right), & k=1, \\
z^{(1) \frac{k-1}{K}}(x, t), & k \geq 2
\end{array}\right\}, \quad(x, t) \in \bar{G}_{h}\left(t^{n}\right) \backslash \bar{G}_{h}^{k}\left(t^{n}\right)\right\} \\
& \text { for }(x, t) \in \bar{G}_{h}\left(t^{n}\right), k=1, \ldots, K, t^{n} \in \bar{\omega}_{0} \text {; } \\
& z^{(1)}(x, t)=z^{(1) \frac{K}{K}}(x, t), \quad(x, t) \in \bar{G}_{h}\left(t^{n}\right), t^{n} \in \bar{\omega}_{0} .
\end{aligned}
$$

Here

$$
\bar{z}^{(1)}\left(x, t ; t^{n}\right)=\bar{v}^{(1)}\left(x, t ; t^{n}\right)=\bar{v}_{(6.3 e)}\left(x, t ; t^{n}\right), \quad(x, t) \in \bar{G}_{h}\left(t^{n}\right), t^{n} \in \bar{\omega}_{0} .
$$

Now we find $z^{(2) \frac{k}{K}}(x, t)$ for $(x, t) \in \bar{G}_{h}\left(t^{n}\right)$, solving the corrected problem

$$
\begin{aligned}
& \Lambda_{(8.3)}\left(z^{(2) \frac{k}{K}}(x, t)\right)=0, \quad(x, t) \in G_{h}^{k}\left(t^{n}\right), \\
& z^{(2) \frac{k}{K}}(x, t)=\left\{\begin{array}{ll}
\bar{z}^{(2)}\left(x, t ; t^{n}\right), & k=1, \\
z^{(2) \frac{k-1}{K}}(x, t), & k \geq 2
\end{array}\right\}, \quad(x, t) \in S_{h}^{k}\left(t^{n}\right) \\
& \text { for }(x, t) \in \bar{G}_{h}^{k}\left(t^{n}\right), k=1, \ldots, K, t^{n} \in \bar{\omega}_{0} \text {; } \\
& \left.z^{(2) \frac{k}{K}}(x, t)=\left\{\begin{array}{ll}
z^{(2) \frac{k}{K}}(x, t), & (x, t) \in \bar{G}_{h}^{k}\left(t^{n}\right), \\
\bar{z}^{(2)}\left(x, t ; t^{n}\right), & k=1, \\
z^{(2) \frac{k-1}{K}}(x, t), & k \geq 2
\end{array}\right\}, \quad(x, t) \in \bar{G}_{h}\left(t^{n}\right) \backslash \bar{G}_{h}^{k}\left(t^{n}\right)\right\} \\
& \text { for }(x, t) \in \bar{G}_{h}\left(t^{n}\right), k=1, \ldots, K, t^{n} \in \bar{\omega}_{0} .
\end{aligned}
$$

The function $z^{(2)}(x, t)$ on the strip $\bar{G}_{h}\left(t^{n}\right)$ is defined by the relation

$$
z^{(2)}(x, t)=z^{(2) \frac{K}{K}}(x, t), \quad(x, t) \in \bar{G}_{h}\left(t^{n}\right), t^{n} \in \bar{\omega}_{0} .
$$

Here

$$
\begin{aligned}
\bar{z}^{(2)}\left(x, t ; t^{n}\right) & =\bar{v}^{(2)}\left(x, t ; t^{n}\right), \quad(x, t) \in \bar{G}_{h}\left(t^{n}\right) \\
\bar{v}^{(2)}\left(x, t ; t^{n}\right) & =\bar{v}^{(2)}\left(x, t ; t^{n}, z^{(1)}(\cdot)\right) \\
& =v\left(x, t^{n} ; t^{n}\right)+z^{(1)}\left(x, t^{n+1}\right)-z^{(1)}\left(x, t^{n}\right), \quad(x, t) \in G_{h}\left(t^{n}\right) \\
v\left(x, t ; t^{n}\right) & =v_{(6.3 f)}\left(x, t ; t^{n}\right), \quad(x, t) \in S_{h}\left(t^{n}\right) ;
\end{aligned}
$$




$$
\begin{aligned}
\Lambda_{(8.3)}\left(z^{(2)}(x, t)\right) \equiv & \Lambda_{(3.2)} z^{(2)}(x, t)-f^{(2)}(x, t), \quad(x, t) \in G_{h}^{k}, \\
f^{(2)}(x, t)= & f^{(2)}\left(x, t ; z^{(1)}(\cdot)\right)=f(x, t) \\
& +\left\{\begin{array}{ll}
p(x, t) 2^{-1} \tau \frac{\partial^{2}}{\partial t^{2}} u(x, 0), \quad t=\tau, \\
p(x, t) 2^{-1} \tau \delta_{2 t} z^{(1)}(x, t), & t \geq 2 \tau
\end{array}\right\}, \quad(x, t) \in G_{h}^{k} .
\end{aligned}
$$

We call the function $z_{(8.3)}^{(2)}(x, t)$ the solution of the domain decomposition - defect correction scheme. The difference scheme (8.3), (4.1) can be briefly written in the operator form

$$
\begin{array}{ll}
Q_{(6.3)}\left(z^{(1)}(x, t) ; f^{(1)}(\cdot), \varphi(\cdot), \psi^{(1)}(\cdot)\right)=0, & (x, t) \in \bar{G}_{h}, \\
Q_{(6.3)}\left(z^{(2)}(x, t) ; f^{(2)}(\cdot), \varphi(\cdot), \psi^{(2)}(\cdot)\right)=0, & (x, t) \in \bar{G}_{h},
\end{array}
$$

where

$$
\begin{aligned}
& f^{(1)}(x, t)=f(x, t), f^{(2)}(x, t)=f_{(8.3 c)}^{(2)}\left(x, t ; z^{(1)}(\cdot)\right), \psi^{(1)}\left(x, t ; t^{n}\right) \equiv 0, \\
& \psi^{(2)}\left(x, t ; t^{n}\right)=\psi^{(2)}\left(x, t ; t^{n}, z^{(1)}(\cdot)\right)=z^{(1)}\left(x, t^{n+1}\right)-z^{(1)}\left(x, t^{n}\right), \\
& (x, t) \in G_{h}\left(t^{n}\right), t=t^{n+1} .
\end{aligned}
$$

For the solutions of difference scheme (8.3), (4.1) the estimate (7.6) remains valid (under assumption that condition (5.6) and the hypotheses of Theorem 7.1 are fulfilled).

8.1.2. In the case of $P>1$ we discretise the process (5.9), (5.8). In the grid constructions (8.2a) and (8.3a), when we solve the finite difference boundary problems on $G_{h}^{k}$, the functions $z^{(1) \frac{k}{K}}(x, t)$ and $z^{(2) \frac{k}{K}}(x, t)$ are replaced by the functions $z_{p}^{(1) \frac{k}{K}}(x, t)$ and $z_{p}^{(2) \frac{k}{K}}(x, t)$, and the set $G_{h}^{k}$ is replaced by the set $G_{p h}^{k}$

$$
\begin{aligned}
& \Lambda_{(8.4)}^{(i)}\left(z_{p}^{(i) \frac{k}{K}}(x, t)\right)=0, \quad(x, t) \in G_{p h}^{k}\left(t^{n}\right), \\
& z_{p}^{(i) \frac{k}{K}}(x, t)=\left\{\begin{array}{ll}
\bar{z}^{(i)}\left(x, t ; t^{n}\right), & k=1, \\
z^{(i) \frac{k-1}{K}}(x, t), & k \geq 2
\end{array}\right\}, \quad(x, t) \in S_{p h}^{k}\left(t^{n}\right), p=1, \ldots, P \\
& \text { for }(x, t) \in \bar{G}_{p h}^{k}\left(t^{n}\right), k=1, \ldots K, t^{n} \in \bar{\omega}_{0}, i=1,2 \text {; } \\
& \left.z^{(i) \frac{k}{K}}(x, t)=\left\{\begin{array}{ll}
z_{p}^{(i) \frac{k}{K}}(x, t), & (x, t) \in \bar{G}_{p h}^{k}\left(t^{n}\right), p=1, \ldots, P, \\
\bar{z}^{(i)}\left(x, t ; t^{n}\right), & k=1, \\
z^{(i) \frac{k-1}{K}}(x, t), & k \geq 2
\end{array}\right\}, \quad(x, t) \in \bar{G}_{h}\left(t^{n}\right) \backslash \bigcup_{p=1}^{P} \bar{G}_{p h}^{k}\left(t^{n}\right)\right\} \\
& \text { for }(x, t) \in \bar{G}_{h}\left(t^{n}\right), k=1, \ldots, K, t^{n} \in \bar{\omega}_{0}, i=1,2 \text {; }
\end{aligned}
$$




$$
z^{(i)}(x, t)=z^{(i) \frac{k}{K}}(x, t), \quad(x, t) \in \bar{G}_{h}\left(t^{n}\right), \quad t^{n} \in \bar{\omega}_{0}, \quad i=1,2 .
$$

Here

$$
\begin{gathered}
\bar{z}^{(i)}\left(x, t ; t^{n}\right)=\bar{v}^{(i)}\left(x, t ; t^{n}\right), \quad(x, t) \in \bar{G}_{h}\left(t^{n}\right), \\
\bar{v}^{(i)}\left(x, t ; t^{n}\right)=\bar{v}^{(i)}\left(x, t ; t^{n},(\cdot)\right) \\
=\left\{\begin{array}{l}
v\left(x, t^{n} ; t^{n}\right)+\psi^{(i)}\left(x, t ; t^{n}\right), \quad(x, t) \in G_{h}\left(t^{n}\right), \\
v\left(x, t ; t^{n}\right), \quad(x, t) \in S_{h}\left(t^{n}\right)
\end{array}\right\}, \quad(x, t) \in \bar{G}_{h}\left(t^{n}\right) ; \\
v\left(x, t ; t^{n}\right)=v_{(6.3 f)}\left(x, t ; t^{n}\right), \quad(x, t) \in S_{h}\left(t^{n}\right), \\
\psi^{(1)}\left(x, t ; t^{n}\right) \equiv 0, \psi^{(2)}\left(x, t ; t^{n}\right)=\psi_{(8.3)}^{(2)}\left(x, t ; t^{n}, z_{(8.4)}^{(1)}(\cdot)\right), \\
\Lambda_{(8.4)}^{(i)}\left(z_{p}^{(i) \frac{k}{K}}(x, t)\right) \equiv G_{(3.2)} z_{p}^{(i) \frac{k}{K}}(x, t)-f^{(i)}(x, t), \quad(x, t) \in G_{p h}^{k}, \\
f^{(1)}(x, t)=f(x, t), f^{n+1} ; \\
f_{(8)}^{(2)}(x, t)=f_{(8.3)}^{(2)}\left(x, t ; z_{(8.4)}^{(1)}(\cdot)\right) .
\end{gathered}
$$

We rewrite the difference scheme (8.4), (6.2) in the operator form

$$
\begin{aligned}
& Q_{(6.5)}\left(z^{(1)}(x, t) ; f^{(1)}(\cdot), \varphi(\cdot), \psi^{(1)}(\cdot)\right)=0, \quad(x, t) \in \bar{G}_{h}, \\
& Q_{(6.5)}\left(z^{(2)}(x, t) ; f^{(2)}(\cdot), \varphi(\cdot), \psi^{(2)}(\cdot)\right)=0, \quad(x, t) \in \bar{G}_{h},
\end{aligned}
$$

where

$$
\begin{gathered}
f^{(1)}(x, t)=f(x, t), f^{(2)}(x, t)=f_{(8.3)}^{(2)}\left(x, t ; z_{(8.4)}^{(1)}(\cdot)\right) \\
\psi^{(1)}\left(x, t ; t^{n}\right) \equiv 0, \psi^{(2)}\left(x, t ; t^{n}\right)=\psi_{(8.3)}^{(2)}\left(x, t ; t^{n}, z_{(8.4)}^{(1)}(\cdot)\right) .
\end{gathered}
$$

Following the arguments given in $[5,6,10]$ the following theorem can be derived.

Theorem 8.1. Let the boundary value problem (2.1) and its solution satisfy the assumptions of Theorem 7.1. Then, under condition (5.6), the solutions of the difference schemes (8.3), (4.1) and (8.4), (4.1) converge, as $N, N_{0} \rightarrow \infty$, to the solution of the boundary value problem \&-uniformly (the solutions of schemes (8.3), (3.1) and (8.4), (3.1) converge for a fixed value of the parameter $\varepsilon)$. For the solutions of the difference schemes on the mesh (4.1) the estimate (7.6) holds. 


\subsection{The Difference Schemes of Third-Order Accuracy in $\tau$}

8.2.1. We approximate the boundary value problem by the alternating scheme with one processor

$$
\begin{aligned}
& \Lambda_{(8.5)}^{(i)}\left(z^{(i) \frac{k}{K}}(x, t)\right)=0, \quad(x, t) \in G_{h}^{k}\left(t^{n}\right), \\
& z^{(i) \frac{k}{K}}(x, t)=\left\{\begin{array}{ll}
\bar{z}^{(i)}\left(x, t ; t^{n}\right), & k=1, \\
z^{(i) \frac{k-1}{K}}(x, t), & k \geq 2
\end{array}\right\}, \quad(x, t) \in S_{h}^{k}\left(t^{n}\right) \\
& \text { for }(x, t) \in \bar{G}_{h}^{k}\left(t^{n}\right), k=1, \ldots, K, t^{n} \in \bar{\omega}_{0}, i=1,2,3 \text {; } \\
& \left.z^{(i) \frac{k}{K}}(x, t)=\left\{\begin{array}{ll}
z^{(i) \frac{k}{K}}(x, t), & (x, t) \in \bar{G}_{p h}^{k}\left(t^{n}\right), \\
\bar{z}^{(i)}\left(x, t ; t^{n}\right), & k=1, \\
z^{(i) \frac{k-1}{K}}(x, t), & k \geq 2
\end{array}\right\}, \quad(x, t) \in \bar{G}_{h}\left(t^{n}\right) \backslash \bar{G}_{h}^{k}\left(t^{n}\right)\right\} \\
& \text { for }(x, t) \in \bar{G}_{h}\left(t^{n}\right), k=1 \ldots, K, t^{n} \in \bar{\omega}_{0}, i=1,2,3 \text {; } \\
& z^{(i)}(x, t)=z^{(i) \frac{K}{K}}(x, t),(x, t) \in \bar{G}_{h}\left(t^{n}\right), t^{n} \in \bar{\omega}_{0}, i=1,2,3 .
\end{aligned}
$$

Here

$$
\begin{aligned}
& \bar{z}^{(i)}\left(x, t ; t^{n}\right)=\bar{v}_{(8.4)}^{(i)}\left(x, t ; t^{n}, \psi^{i}(\cdot)\right), \quad(x, t) \in \bar{G}_{h}\left(t^{n}\right), i=1,2,3 ; \\
& \bar{\psi}^{(s)}\left(x, t ; t^{n}\right)=\bar{\psi}_{(8.4)}^{(s)}\left(x, t ; t^{n}\right), \quad s=1,2, \\
& \bar{\psi}^{(3)}\left(x, t ; t^{n}\right)=z^{(2)}\left(x, t^{n+1}\right)-z^{(2)}\left(x, t^{n}\right) ; \\
& \Lambda_{(8.5)}^{(s)}\left(z^{(s) \frac{k}{K}}(x, t)\right)=\Lambda_{(8.4)}^{(s)}\left(z^{(s) \frac{k}{K}}(x, t)\right), \quad s=1,2, \\
& \Lambda_{(8.5)}^{(3)}\left(z^{(3) \frac{k}{K}}(x, t)\right)=\Lambda_{(3.2)} z^{(3) \frac{k}{K}}(x, t)-f^{(3)}(x, t), \\
& f^{(3)}(x, t)=f^{(3)}\left(x, t ; z^{(1)}(\cdot), z^{(2)}(\cdot)\right) \\
& =f(x, t)+\left\{\begin{array}{cc}
p(x, t)\left(C_{11} \tau \frac{\partial^{2}}{\partial t^{2}} u(x, 0)+C_{12} \tau^{2} \frac{\partial^{3}}{\partial t^{3}} u(x, 0)\right), & t=\tau, \\
p(x, t)\left(C_{21} \tau \frac{\partial^{2}}{\partial t^{2}} u(x, 0)+C_{22} \tau^{2} \frac{\partial^{3}}{\partial t^{3}} u(x, 0)\right), & t=2 \tau, \\
p(x, t)\left(C_{31} \tau \delta_{2 \bar{t}} z^{(2)}(x, t)+C_{32} \tau^{2} \delta_{3 \bar{t}} z^{(1)}(x, t)\right), & t \geq 3 \tau
\end{array}\right\},
\end{aligned}
$$




$$
(x, t) \in G_{h}^{k}, C_{i j}=C_{i j(7.7)}
$$

8.2.2. In the case of $P>1$ processors we use the scheme

$$
\begin{aligned}
& \Lambda_{(8.5)}^{(i)}\left(z_{p}^{(i) \frac{k}{K}}(x, t)\right)=0, \quad(x, t) \in G_{p h}^{k}\left(t^{n}\right), \\
& z_{p}^{(i) \frac{k}{K}}(x, t)=\left\{\begin{array}{ll}
\bar{z}^{(i)}\left(x, t ; t^{n}\right), & k=1, \\
z^{(i) \frac{k-1}{K}}(x, t), & k \geq 2
\end{array}\right\}, \quad(x, t) \in S_{p h}^{k}\left(t^{n}\right), p=1, \ldots, P \\
& \text { for }(x, t) \in \bar{G}_{p h}^{k}\left(t^{n}\right), k=1, \ldots, K, t^{n} \in \bar{\omega}_{0}, i=1,2,3 \text {; } \\
& \left.z^{(i) \frac{k}{K}}(x, t)=\left\{\begin{array}{cc}
z_{p}^{(i) \frac{k}{K}}(x, t), \quad(x, t) \in \bar{G}_{p h}^{k}\left(t^{n}\right), p=1, \ldots, P, \\
\bar{z}^{(i)}\left(x, t ; t^{n}\right), \quad k=1, \\
z^{(i) \frac{k-1}{K}}(x, t), \quad k \geq 2
\end{array}\right\}, \quad(x, t) \in \bar{G}_{h}\left(t^{n}\right) \backslash \cup_{p=1}^{P} \bar{G}_{p h}^{k}\left(t^{n}\right)\right\} \\
& \text { for }(x, t) \in \bar{G}_{h}\left(t^{n}\right), k=1, \ldots, K, t^{n} \in \bar{\omega}_{0}, i=1,2,3 \text {; } \\
& z^{(i)}(x, t)=z^{(i) \frac{K}{K}}(x, t), \quad(x, t) \in \bar{G}_{h}\left(t^{n}\right), t^{n} \in \bar{\omega}_{0}, i=1,2,3 .
\end{aligned}
$$

Here

$$
\begin{gathered}
\bar{z}^{(i)}\left(x, t ; t^{n}\right)=\bar{v}_{(8.4)}^{(i)}\left(x, t ; t^{n}, \psi^{i}(\cdot)\right), \quad(x, t) \in \bar{G}_{h}\left(t^{n}\right), \\
\psi^{(i)}\left(x, t ; t^{n}\right)=\psi_{(8.5)}^{(i)}\left(x, t ; t^{n}\right)=\psi_{(8.5)}^{i}\left(x, t ; t^{n}, z_{(8.6)}^{(j)}(\cdot), 0<j<i\right), \\
f^{(i)}(x, t)=f_{(8.5)}^{(i)}\left(x, t ; z_{(8.6)}^{(j)}(\cdot), 0<j<i\right) .
\end{gathered}
$$

Theorem 8.2. Let the boundary value problem (2.1) and its solution satisfy the assumptions of Theorem 7.2. Then, under condition (5.6), the solutions of the difference schemes (8.5), (4.1) and (8.6), (4.1) converge, as $N, N_{0} \rightarrow \infty$, to the solution of the boundary value problem $\varepsilon$-uniformly (the solutions of difference schemes (8.5), (3.1) and (8.6), (3.1) converge for a fixed value of the parameter $\varepsilon)$. For the solutions of the difference schemes the estimate (7.9) holds.

The proof of Theorems 8.1, 8.2 can be done by using the technique from $[5,6,10]$. In a similar way one can construct parallel schemes with an arbitrary high order of time-accuracy $\mathcal{O}\left(N^{-1} \ln N+\tau^{l}\right), l>3$. 


\section{Conclusion}

In order to efficiently solve a singularly perturbed parabolic PDE by an $\varepsilon$-uniform discretisation procedure, 1st order accurate in space and high-order in time, we have studied a defect correction procedure. To possibly reduce the computation time, we have splitted the procedure in $P$ independent processes, preserving $\varepsilon$-uniform convergence. The requirements are precisely described under which the splitting does not affect the accuracy of the method. Note that such a technique to construct high-order time-accurate parallel methods can be naturally extended to multidimensional convection-dominated problems without affecting the accuracy.

\section{Appendix: Estimates of the Solution and its Derivatives}

Here we rely on the a-priori estimates for the solution of problem (2.1) and its derivatives as derived for elliptic and parabolic equations in [9].

We denote by $H^{(\alpha)}(\bar{G})=H^{\alpha, \alpha / 2}(\bar{G})$ the Hölder space, where $\alpha$ is an arbitrary positive number [15]. We suppose that the functions $f(x, t)$ and $\varphi(x, t)$ satisfy compatibility conditions at the corner points so that the solution of the boundary value problem is smooth for every fixed value of the parameter $\varepsilon$.

For simplicity, the following conditions are assumed to hold at the corner points $S_{0} \cap \bar{S}^{L}$

$$
\begin{gathered}
\frac{\partial^{k}}{\partial x^{k}} \varphi(x, t)=\frac{\partial^{k_{0}}}{\partial t^{k_{0}}} \varphi(x, t)=0, \quad k+2 k_{0} \leq[\alpha]+2 n, \\
\frac{\partial^{k+k_{0}}}{\partial x^{k} \partial t^{k_{0}}} f(x, t)=0, \quad k+2 k_{0} \leq[\alpha]+2 n-2,
\end{gathered}
$$

where $[\alpha]$ is the integer part of a number $\alpha, \alpha>0, n \geq 0$ is an integer number. We also suppose that $[\alpha]+2 n \geq 4$.

Using interior a-priori estimates and estimates up to the boundary for the regular function $\tilde{u}(\xi, \eta)$, see [15], where $\tilde{u}(\xi, \eta)=u(x(\xi), t), \xi=x / \varepsilon, \eta=t / \varepsilon$, we obtain

$$
\left|\frac{\partial^{k+k_{0}}}{\partial x^{k} \partial t^{k_{0}}} u(x, t)\right| \leq M \varepsilon^{-k-k_{0}},(x, t) \in \bar{G}, \quad k+2 k_{0} \leq 2 n+4, n \geq 0 .
$$

This estimate holds, for example, for

$$
u \in H^{(2 n+4+v)}(\bar{G}), \quad v>0,
$$

where $v$ is some small number. For example, (9.3) is guaranteed for the solution of problem (2.1) if $a, b, c, p, f \in H^{(\alpha+2 n-2)}(\bar{G}), \varphi \in H^{(\alpha+2 n)}(\bar{G}), \alpha>4, n \geq 0$ and condition (9.1) is fulfilled. 
In fact we need a more accurate estimate than (9.2). Therefore, we decompose the solution of problem (2.1) into a regular component $U(x, t)$ and a singular component $W(x, t)$

$$
u(x, t)=U(x, t)+W(x, t), \quad(x, t) \in \bar{G},
$$

where $W(x, t)$ represents the regular boundary layer. The function $U(x, t)$ is the smooth solution of (2.1a) satisfying condition (2.1b) on $S \backslash S_{0}^{L}$. For example, under suitable assumptions for the data of the problem, we can consider the solution of the Dirichlet boundary value problem for (2.1a) smoothly extended to the domain $\bar{G}^{*}\left(\bar{G}^{*}\right.$ is a sufficiently large neighbourhood of $\bar{G}$ on the left of the set $S_{0}^{L}$ ). On the domain $\bar{G}$ the coefficients and the initial value of the extended problem are the same as for (2.1). Then the function $U(x, t)$ is the restriction (on $\bar{G}$ ) of the solution to the extended problem, and $U \in H^{(4+2 n+v)}(\bar{G}), \quad v>0$. The function $W(x, t)$ is the solution of Dirichlet's problem for the parabolic equation

$$
L_{(2.1)} W(x, t)=0, \quad(x, t) \in G, \quad W(x, t)=u(x, t)-U(x, t), \quad(x, t) \in S .
$$

If (9.3) is true then $U, W \in H^{(4+2 n+v)}(\bar{G})$.

We suppose that $a, b, c, p, f \in H^{(2+\alpha+4 n)}(\bar{G}), \varphi \in H^{(2+\alpha+4 n)}(\bar{G}), \alpha>4, n \geq 0$. Now, for the functions $U(x, t)$ and $W(x, t)$ we derive the estimates

$$
\begin{gathered}
\left|\frac{\partial^{k+k_{0}}}{\partial x^{k} \partial t^{k_{0}}} U(x, t)\right| \leq M\left[1+\varepsilon^{2+n-k-k_{0}}\right], \\
\left|\frac{\partial^{k+k_{0}}}{\partial x^{k} \partial t^{k_{0}}} W(x, t)\right| \leq M\left[1+\varepsilon^{2+n-k-k_{0}}\right] \varepsilon^{-k} \exp \left(-m_{(9.6)} \varepsilon^{-1} x\right), \\
(x, t) \in \bar{G}, \quad k+2 k_{0} \leq 2 n+4,
\end{gathered}
$$

where $m_{(9.6)}$ is an arbitrary number from the interval $\left(0, m_{0}\right), m_{0}=$ $\min _{\bar{G}}\left[a^{-1}(x, t) b(x, t)\right]$. The estimates (9.5) and (9.6) hold, for example, when $a, b, c$, $p, f \in H^{(2+\alpha+4 n)}(\bar{G}), \varphi \in H^{(2+\alpha+4 n)}(\bar{G})$ and

$$
U, W \in H^{(4+2 n+v)}(\bar{G}), \quad v>0 .
$$

The inclusions (9.7) are guaranteed if $a, b, c, p, f \in H^{(\alpha+2 n-2)}(\bar{G}), \varphi \in H^{(\alpha+2 n)}(\bar{G})$, $\alpha>4, n \geq 0$ and condition (9.3) is fulfilled. We summarize these results in the following theorem.

Theorem 9.1. Assume in (2.1) that $a, b, c, p, f \in H^{2+\alpha+4 n}(\bar{G}), \varphi \in H^{(2+\alpha+4 n)}(\bar{G})$, $\alpha>4, n \geq 0$ and let condition (9.3) be fulfilled. Then, for the solution $u(x, t)$ of problem (2.1), and for its components in representation (9.4), it follows that $u, U$, $W \in H^{(4+2 n)}(\bar{G})$ and that the estimates (9.2), (9.5), (9.6) hold. 


\section{References}

[1] Farrell, P. A., Hemker, P. W., Shishkin, G. I.: Discrete approximations for singularly perturbed boundary value problems with parabolic layers, I. J. Comput. Math. 14, 71-97 (1996).

[2] Farrell, P. A., Hemker, P. W., Shishkin, G. I.: Discrete approximations for singularly perturbed boundary value problems with parabolic layers, II. J. Comput. Math. 14, 183-194 (1996).

[3] Farrell, P. A., Hemker, P. W., Shishkin, G. I.: Discrete approximations for singularly perturbed boundary value problems with parabolic layers, III. J. Comput. Math. 14, 273-290 (1996).

[4] Farrell, P. A., Miller, J. J. H., O’Riordan, E., Shishkin, G. I.: A uniformly convergent finite difference scheme for a singularly perturbed semilinear equation. SIAM J. Numer. Anal. 33, 1135-1149 (1996).

[5] Hemker, P. W., Shishkin, G. I., Shishkina, L. P.: The use of defect correction for the solution of parabolic singular perturbation problems. ZAMM 77, 59-74 (1997).

[6] Hemker, P. W., Shishkin, G. I., Shishkina, L. P.: $\varepsilon$-uniform schemes with high-order timeaccuracy for parabolic singular perturbation problems. IMA J. Numer. Anal. 20, 99-121 (2000).

[7] Miller, J. J. H., O'Riordan, E., Shishkin, G. I.: Fitted numerical methods for singular perturbation problems. Singapore: World Scientific, 1996.

[8] Roos, H.-G., Stynes, M., Tobiska, L.: Numerical methods for singularly perturbed differential equations. Berlin, Heidelberg, New York, Tokyo: Springer, 1996.

[9] Shishkin, G. I.: Grid approximation of singularly perturbed elliptic and parabolic equations. Ekaterinburg: Ural Branch of Russian Acad. Sci. 1992 [in Russian].

[10] Shishkin, G. I.: Acceleration of the process of the numerical solution to singularly perturbed boundary value problems for parabolic equations on the basis of parallel computations. Russ. J. Numer. Anal. Math. Modelling 12, 271-291 (1997).

[11] Samarsky, A. A.: Theory of difference schemes. Moscow: Nauka, 1989 [in Russian].

[12] Shishkin, G. I.: Approximation of solutions of singularly perturbed boundary value problems with a parabolic boundary layer. USSR Comput. Math. Math. Phys. 29, 1-10 (1989).

[13] Shishkin, G. I.: Grid approximation of singularly perturbed elliptic equation in domain with characteristic bounds. Sov. J. Numer. Anal. Math. Modell. 5, 327-343 (1990).

[14] Il'in, A. M., Kalashnikov, A. S., Oleinik, O. A.: Linear second-order equations of parabolic type. Uspekhi Mat. Nauk 17, 3-146 (1962) [in Russian].

[15] Ladyzhenskaya, O. A., Solonnikov, V. A., Ural'tseva, N. N.: Linear and Quasi-linear Equations of Parabolic Type. Transl. of Math. Monographs 23, AMS, Providence, RI 1968.

[16] Shishkin, G. I.: Grid approximation of certain singularly perturbed problems of convective transfer with diffusion. Sov. J. Numer. Anal. Math. Modell. 6, 395-420 (1991).

\author{
Pieter W. Hemker \\ CWI \\ Kruislaan 413 \\ NL-1098 SJ Amsterdam \\ The Netherlands \\ e-mail: pieth@cwi.nl
}

Grigorii I. Shishkin, Lidia P. Shishkina Institute of Mathematics and Mechanics Ural Branch of Russian Academy of Science 620219 Ekaterinburg

Russia

e-mails: Grigorii@shishkin.ural.ru Lida@shishkin.ural.ru 